\title{
Resonant four-wave mixing of laser radiation in plasmas
}

\author{
A. Lal and C. Joshi \\ Department of Electrical Engineering, University of California, Los Angeles, Los Angeles, California 90024
}

Received December 13, 1990; revised manuscript received April 8, 1991

\begin{abstract}
Experimental evidence of resonant four-wave mixing of $\mathrm{CO}_{2}$ laser radiation in a plasma is presented for the first time to our knowledge. Comparison of the experiment with theory indicates that, while collisions lead to a narrow spectral width of the ion acoustic resonance, convection and detuning owing to laser heating limit the enhancement of the signal reflectivity to below the expected value.
\end{abstract}

\section{INTRODUCTION}

Optical phase conjugation is an area of nonlinear optics with a wide variety of potential applications. ${ }^{1,2}$ One method of generating a phase-conjugate signal is with four-wave mixing (FWM). In FWM three input beams interact in a nonlinear medium and a fourth beam is produced that is the phase conjugate of one of the input waves. Degenerate four-wave mixing (DFWM) is a special case of FWM in which all the beams are at the same frequency. $^{3}$ In a plasma DFWM is an effective technique for phase conjugation in high-density, low-temperature plasmas. ${ }^{4-6}$ One method of enhancing the phase-conjugate signal over and above the DFWM level is with resonant four-wave mixing (RFWM), in which two of the input beams beat at a plasma resonance. ${ }^{7-10}$ In addition to enhancing the generated wave, RFWM can also serve as a diagnostic for many plasma parameters, such as electron and ion temperatures, ion acoustic velocity, and damping rate. In this paper experimental evidence of RFWM in plasmas with $\mathrm{CO}_{2}$ laser radiation $(10.6 \mu \mathrm{m})$ is presented and data are compared with theoretical predictions.

\section{DEGENERATE FOUR-WAVE MIXING}

\section{Experimental Setup}

The experimental setup for DFWM is shown in Ref. 6 . The $\mathrm{CO}_{2}$ laser produces a gain-switched, singlelongitudinal-mode, 150-ns FWHM pulse, with an energy of approximately $200 \mathrm{~mJ}$. After the beam leaves the oscillator, a telescope is used to expand the width of the beam from 0.7 to $3.5 \mathrm{~cm}$. In DFWM the main beam is then split into three parts, first with a $10 \%$ beam splitter and then with a $50 \%$ beam splitter. The three beams are focused and converge in a 1-mm spot in the center of the plasma. The two strong pump beams are counterpropagating, while the weaker probe beam enters at a slight angle with respect to the forward pump. The plasma is produced by a high current arc discharge in a few Torr of gas (argon, nitrogen, helium, or hydrogen). Some typical parameters for the plasma are a density of $n_{0}=10^{17} \mathrm{~cm}^{-3}$ and electron and ion temperatures of $T_{e}=T_{i}=2 \mathrm{eV}$. The phase-conjugate signal is generated at the center of the plasma and retraces the path of the probe beam. 'The signal is then split with a $50 \%$ beam splitter, and its intensity is measured with a liquid-helium-cooled $\mathrm{Cu}: \mathrm{Ge}$ detec- tor. A retroreflecting mirror may be placed in the path of the probe beam to simulate the phase-conjugate signal. This also provides a reference intensity for the probe beam. The reflectivity is defined as the ratio of signal intensity to probe intensity.

\section{Ponderomotive Force}

FWM is a nonlinear process that is dependent on the material's third-order susceptibility, $\chi^{(3)}$. In a plasma the susceptibility is related to the density modulations produced by the laser radiation. In the case of DFWM two mechanisms can produce these density modulations: the ponderomotive force and the thermal force. The ponderomotive force is proportional to the gradient of the laser's intensity and produces a periodic modulation in the density. The third-order susceptibility $\chi^{(3)}$ that is due to the ponderomotive force has been calculated by Steel and $\mathrm{Lam}^{4}$ :

$$
\chi^{(3)}=\frac{n_{0} / n_{c}{ }^{2}}{(8 \pi)^{2}\left(T_{e}+T_{i}\right)} .
$$

Here $n_{0}$ is the plasma electron density, $n_{c}=m w_{0}^{2} / 4 \pi e^{2}$ is the critical density of the plasma, and $T_{e}\left(T_{i}\right)$ is the plasma electron (ion) temperature. The theoretical value for the reflectivity is given by

$$
R=\left|\frac{2 \pi w_{0}}{c} \chi^{(3)} I L\right|^{2},
$$

where $I$ is the laser intensity and $L$ is the interaction length. If $\chi^{(3)}$ is determined by the ponderomotive force, then the reflectivity should scale as

$$
R \propto \frac{n_{0}^{2} \lambda^{6}}{T_{e}^{2}} .
$$

Another method of visualizing FWM in a plasma is by using a grating picture. Two of the waves interfere to form a density grating, and the third beam Bragg scatters off this grating. For example, if the angle between the forward pump $f$ and the probe $p$ is small, then the forward pump and the probe interfere to form a stationary, longwavelength grating, and the backward pump scatters off this. Similarly, the backward pump $b$ and the probe $p$ form a stationary, short-wavelength grating for the 


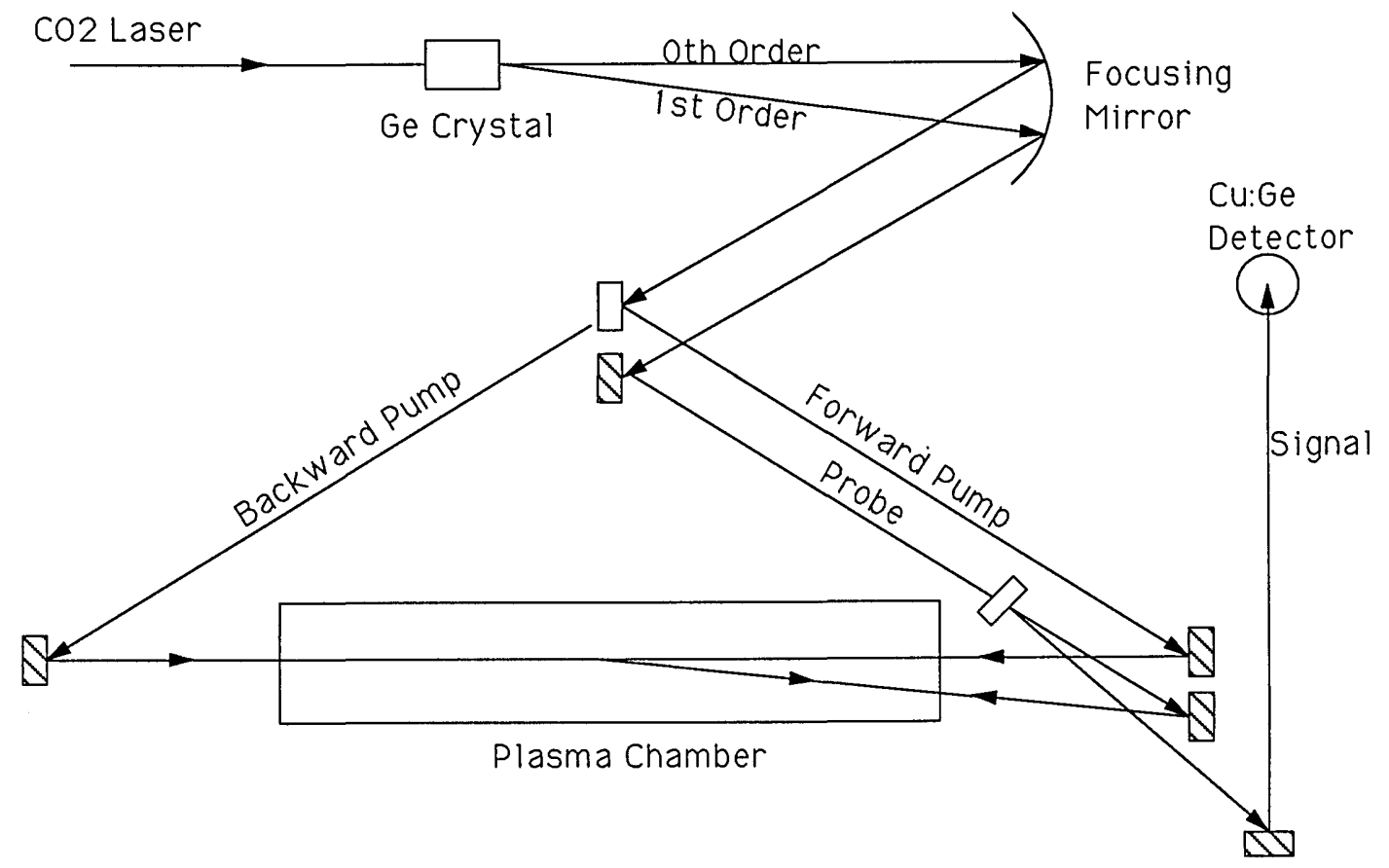

Mirror

\section{0\% Beam Splitter}

Fig. 1. Experimental setup for RFWM.

forward pump. The reflectivity can be rewritten with the Bragg scattering formula

$$
R=C\left|\frac{\pi}{2} \frac{n_{0}}{n_{c}} \frac{n_{1}}{n_{0}} \frac{L}{\lambda}\right|^{2},
$$

where $C$ is the ratio of the pump intensity to the probe intensity and the density perturbation level is

$$
\frac{n_{1}}{n_{0}}=\sum_{i=f, b}\left(\frac{v_{o}}{c}\right)_{i}\left(\frac{v_{o}}{c}\right)_{p} \frac{1}{4} \frac{m c^{2}}{T_{e}+T_{i}}
$$

where $v_{0} / c=e E / m w_{0} c$ is the normalized oscillating velocity of the electrons and $f$ and $b$ refer to the forward and backward pumps, respectively.

\section{Thermal Force}

The two gratings have equal importance when the ponderomotive force is the nonlinear mechanism. When the thermal force is significant, however, this is no longer the case. This was first pointed out by Federici and Mansfield. ${ }^{5}$ Since the plasma heats in proportion to the laser intensity, the laser produces a temperature grating in the plasma. This temperature modulation then produces a density grating to supplement the ponderomotive force. The effects of this thermal force are significant only when the collisional mean free path is shorter than the density grating scale length. Therefore the thermal force is more important for the small- $k$ (long-wavelength) grating. The density perturbation $n_{1} / n_{0}$ now becomes

$$
\frac{n_{1}}{n_{0}}=\sum_{i=f, b}\left(\frac{v_{o}}{c}\right)_{i}\left(\frac{v_{o}}{c}\right)_{p} \frac{1}{4} \frac{m c^{2}}{T_{e}+T_{i}}(1+A),
$$

where $A=n_{0} v_{e} / k_{i p}{ }^{2} \kappa_{e}$ is the thermal enhancement factor. The reflectivity now scales as

$$
R \propto \frac{n_{0}{ }^{6} \lambda^{10}}{T_{e}{ }^{10}} .
$$

Experimental evidence of DFWM was reported by Kitagawa et $a l .^{6}$ The experiments showed that the reflectivity scales as $n_{0}{ }^{6}$, demonstrating the importance of the thermal force. Rotating the polarization of the input beams also demonstrated the importance of the small- $k$ grating.

\section{RESONANT FOUR-WAVE MIXING}

\section{Theory}

The density fluctuations of the plasma gratings, and therefore the reflectivity of FWM, can be further enhanced by the RFWM method. In RFWM the pump beams have identical frequencies $w_{0}$, while the probe beam has a slightly different frequency $w_{p}=w_{0}+w_{m}$, where $w_{m} \ll w_{0}$. The frequency difference between the pumps and the probe is chosen so that $w_{0}-w_{p}=k c_{s}$, where $k$ is the grating wave number and $c_{s}$ is the ion acoustic velocity. When this condition is met, the density modulation is increased by the generation of the ion acoustic wave, whose dispersion relationship is given by

$$
w=k_{\text {ion }} c_{s} \text {. }
$$

For temperatures of $T_{e}=T_{i}=2.25 \mathrm{eV}$, the ion acoustic velocity in an argon plasma is equal to

$$
c_{s}=\sqrt{\frac{Z T_{e}+\gamma T_{i}}{M}}=4.64 \times 10^{5} \mathrm{~cm} / \mathrm{s} .
$$

The wave number for the small- $k$ grating, for a $\mathrm{CO}_{2}$ laser 
with a probe angle of $4^{\circ}$, is $k_{f p}=413.74 \mathrm{~cm}^{-1}$, so the resonant frequency $w_{\text {res }}=k c_{s}=192 \mathrm{MHz}$, or $f_{\text {res }}=w_{\text {res }} / 2 \pi=$ $30.57 \mathrm{MHz}$. If the frequency of the probe beam is shifted with respect to the forward pump by $30.57 \mathrm{MHz}$, the ion acoustic wave can be excited in the plasma.

In our experiment the frequency shift is produced by acousto-optically modulating the single-mode $\mathrm{CO}_{2}$ laser pulse. A transducer sends an acoustic wave through a germanium crystal operating in a standing wave mode. The laser scatters off this wave, and the light in the first diffracted order is both upshifted and downshifted by the acoustic frequency. This first-diffracted-order light now becomes the probe beam (see Fig. 1). The light in the zeroth order is not greatly affected by the acoustic wave and serves as the pump beam. Using this setup, one can shift the frequency of the probe beam from 20 to $60 \mathrm{MHz}$.

An estimate of the increase of the density modulation $n_{1} / n_{0}$ can be made with a simple oscillator model. From the equations of motion and continuity, the equation for the ion acoustic wave in the presence of an external force, in steady state, is given by

$$
\left(w^{2}+j w \gamma-k^{2} c_{s}^{2}\right) n_{1}=\frac{j k F_{\mathrm{NL}}}{M} .
$$

In this equation, $\gamma$ is the damping rate and $F_{\mathrm{NL}}$ is assumed to be the ponderomotive force.

From the definition of the ponderomotive force,

$$
F=\frac{-e^{2}}{2 m w_{0}^{2}} \nabla\left\langle E^{2}\right\rangle
$$

the right-hand side of Eq. (10) can be rewritten as

$$
\frac{-j k}{M} F_{\mathrm{NL}}=\frac{m c^{2} k^{2} c_{s}{ }^{2}}{2\left(T_{e}+T_{i}\right)}\left(\frac{v_{o}}{c}\right)_{f}\left(\frac{v_{o}}{c}\right)_{p} .
$$

From Eq. (5) this becomes

$$
\frac{-j k}{M} F_{\mathrm{NL}}=\left(\frac{n_{1}}{n_{0}}\right)_{\text {pond }} k^{2} c_{s}{ }^{2} .
$$

Therefore the total density modulation (in steady state) is equal to

$$
\left(\frac{n_{1}}{n_{0}}\right)_{\text {tot }}=\left(\frac{n_{1}}{n_{0}}\right)_{\text {pond }} \frac{k^{2} c_{s}^{2}}{w^{2}+j w \gamma-k^{2} c_{s}^{2}} .
$$

When the frequency difference is equal to the ion acoustic frequency, the real part of the denominator vanishes, and the grating strength is equal to

$$
\left(\frac{n_{1}}{n_{0}}\right)_{\text {tot }}=\left(\frac{n_{1}}{n_{0}}\right)_{\text {pond }}\left(\frac{w}{j \gamma}\right) .
$$

If $v_{i i} \gg k c_{s}$, then collisional effects, which give rise to the thermal force, are important. When these thermal forces are included the density modulation becomes

$$
\left(\frac{n_{1}}{n_{0}}\right)_{\text {tot }}=\left(\frac{n_{1}}{n_{0}}\right)_{\text {pond }}(1+A)\left(\frac{w}{j \gamma}\right) .
$$

Since the reflectivity from Eq. (4) is proportional to $\left(n_{1} / n_{0}\right)^{2}$, the reflectivity in RFWM should be increased by a factor of $(w / \gamma)^{2}$. In addition, since the right-hand side of Eq. (14) is Lorentzian in form, the reflectivity as a func- tion of frequency difference should have a Lorentzian shape, where the width of the profile is related to the damping rate $\gamma$.

\section{Experimental Results}

RFWM experiments were performed in three gases: argon, nitrogen, and helium. The first-order diffracted beam from the acousto-optic crystal was used for the probe beam, while the zeroth order was used for the pump beams. The pump beams have a strong component at $w_{0}$ and small components at $w_{0} \pm w_{m}$, while the probe beam has frequencies $w_{0} \pm w_{m}$. Typical pulse shapes of the pump, probe, and signal beams are shown in Fig. 2. For
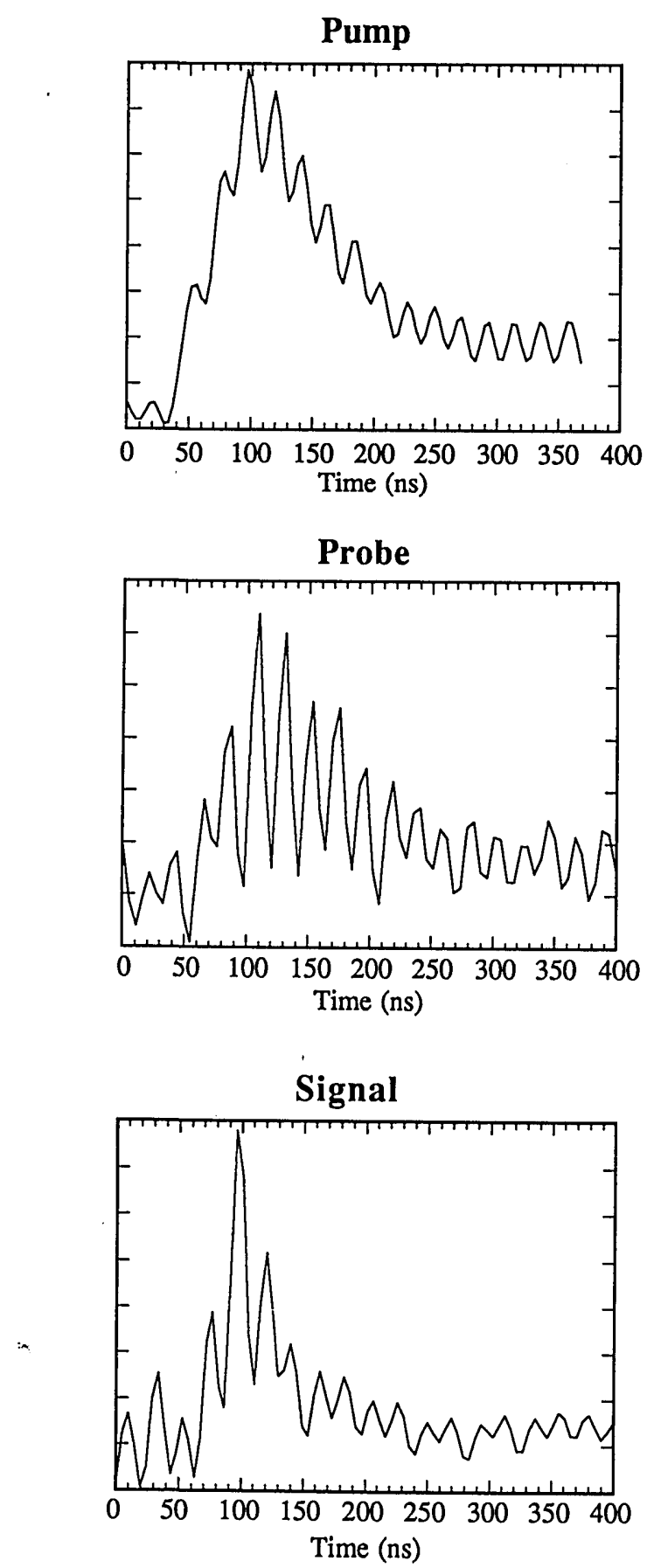

Fig. 2. Typical $\mathrm{CO}_{2}$ laser intensity profiles for the pump, probe, and signal pulses. 
(a)

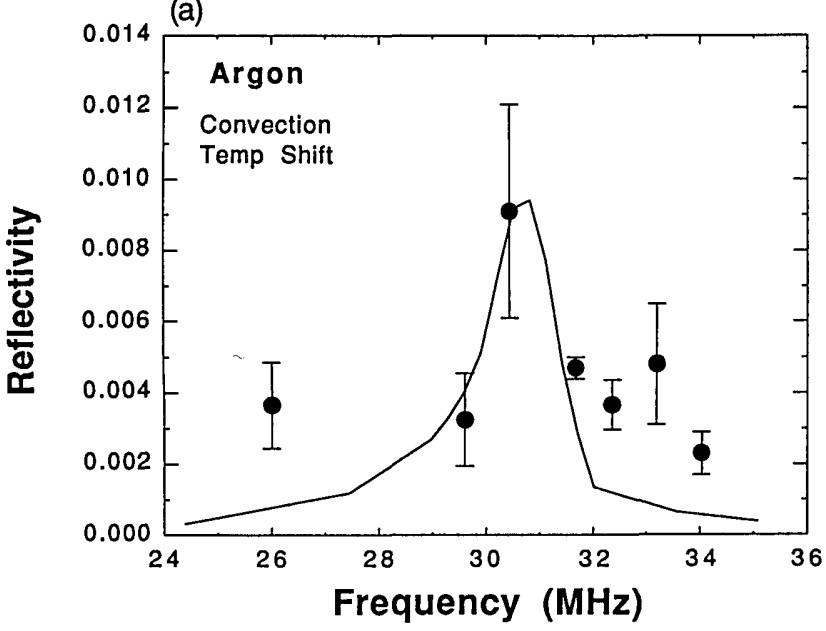

(b)

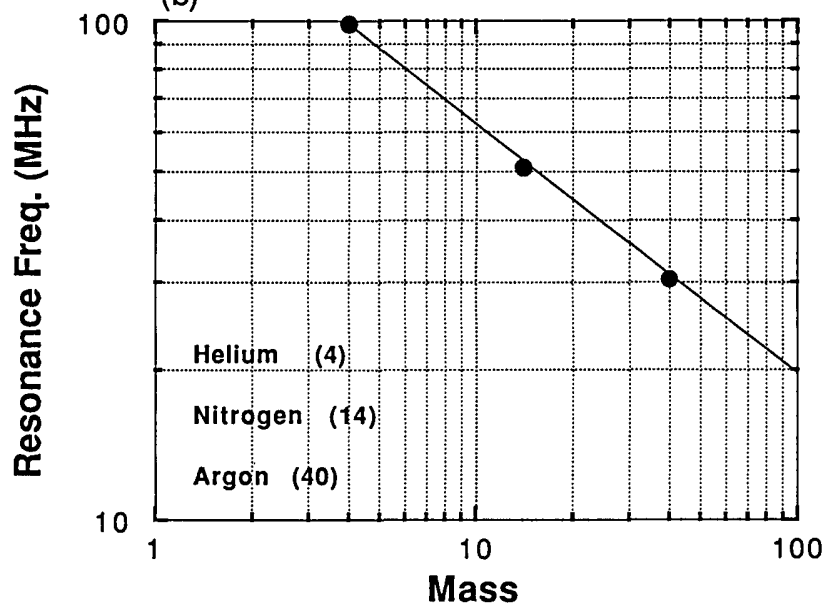

Fig. 3. (a) Experimentally measured reflectivity versus applied modulation frequency for an argon plasma. The solid curve represents a simple theoretical model that is described in the Temperature Shift section. (b) Resonance frequency as a function of mass for three gases: argon, nitrogen, and helium. The experimental points fit very well with the expected $1 / \sqrt{M}$ dependence.

the probe beam, the beating of the frequencies at $w_{0}+w_{m}$ and $w_{0}-w_{m}$ produces an intensity modulation in the pulse at a frequency $w=2 w_{m}$.

For argon the probe beam enters the chamber at an angle of $4^{\circ}$ with respect to the forward pump. The frequency applied to the crystal is varied from 20 to $35 \mathrm{MHZ}$. At each frequency, the peak reflectivity is measured over several shots, and the average peak reflectivity is plotted in Fig. 3(a). (The solid curve is a theoretical prediction explained below.) The plot shows a significant peak at approximately $30.5 \mathrm{MHz}$, then a gradual decrease. Away from resonance, the reflectivity should return to the DFWM value, since all three beams have some components at the same frequency. The enhancement that is due to RFWM seems to be approximately a factor of 4 . The resonance peak is very narrow, with a FWHM of approximately $2 \mathrm{MHz}$.

The experiment was then performed with a nitrogen plasma. The resonance frequency is proportional to the ion acoustic velocity $c_{s}$, which has a $1 / \sqrt{M}$ dependence.
Since the mass of nitrogen is smaller than the mass of argon by a factor of $14 / 40$, the resonance frequency is expected to be greater by $\sqrt{40 / 14}=1.69$. The plot of reflectivity versus frequency for nitrogen shows a peak at approximately $50 \mathrm{MHz}$, with a width of approximately $6 \mathrm{MHz}$. The ratio of the resonance frequency of nitrogen to the resonance frequency of argon is

$$
\frac{f_{\text {res }}\left(\mathrm{N}_{2}\right)}{f_{\text {res }}(\mathrm{Ar})}=\frac{50 \mathrm{MHz}}{30.5 \mathrm{MHz}}=1.64 \text {. }
$$

The shift in frequency agrees well with the theoretical prediction.

The experiment was also performed with a helium plasma. Since helium has a much smaller mass than argon, the frequency shift should be large. Owing to the limits of the crystal modulation frequency, the angle between the probe and the forward pump was reduced from $4^{\circ}$ to $2^{\circ}$. This reduces the wave number $k$ and therefore reduces the resonance frequency. The peak of the helium reflectivity curve is at approximately $49 \mathrm{MHz}$. If we compare this with the theoretical prediction, we get

$$
\frac{f_{\text {res }}(\mathrm{He})}{f_{\text {res }}(\mathrm{Ar})}=\frac{49 \mathrm{MHz} \times 2}{30.5 \mathrm{MHz}}=3.21 \text {. }
$$

The mass ratio is $\sqrt{40 / 4}=3.16$, so this also agrees well with the theory. In Fig. 3(b) the three resonant frequencies are plotted, along with a theoretical $1 / \sqrt{M}$ dependence.

In order to compare the enhancement factor and resonance width of RFWM with the theoretical values, one must determine the factor $w / \gamma$.

\section{DAMPING MECHANISMS}

\section{Landau Damping}

In order to find the theoretical enhancement in reflectivity, it is necessary to find the correct damping rate. For ion acoustic waves, the important damping mechanism generally is ion Landau damping. ${ }^{11}$ The Landau damping rate decreases exponentially with the ratio $\theta=Z T_{e} / T_{i}$. If the temperatures are assumed to be approximately equal, so that $\theta=1$, then the damping rate is approximately

$$
\frac{\operatorname{Im} w}{\operatorname{Re} w}=0.3 .
$$

This implies that the reflectivity should be enhanced by a factor of approximately 10 , while the width of the resonance peak should be quite broad. For argon; with a resonant frequency of $30.5 \mathrm{MHz}$, the damping rate should be $10 \mathrm{MHz}$. This width is much broader than the experimentally measured width.

Landau damping, however, is not the important damping mechanism in this case. The ion-ion collision frequency, which is proportional to the density and inversely proportional to the temperature, is of the order of $10^{10} \mathrm{~s}^{-1}$. The collision frequency is much higher than the acoustic frequency $w_{\text {ac }}$, so Landau damping cannot occur.

\section{Collisional Narrowing}

When the plasma is highly collisional, other damping mechanisms become more important than ion Landau 


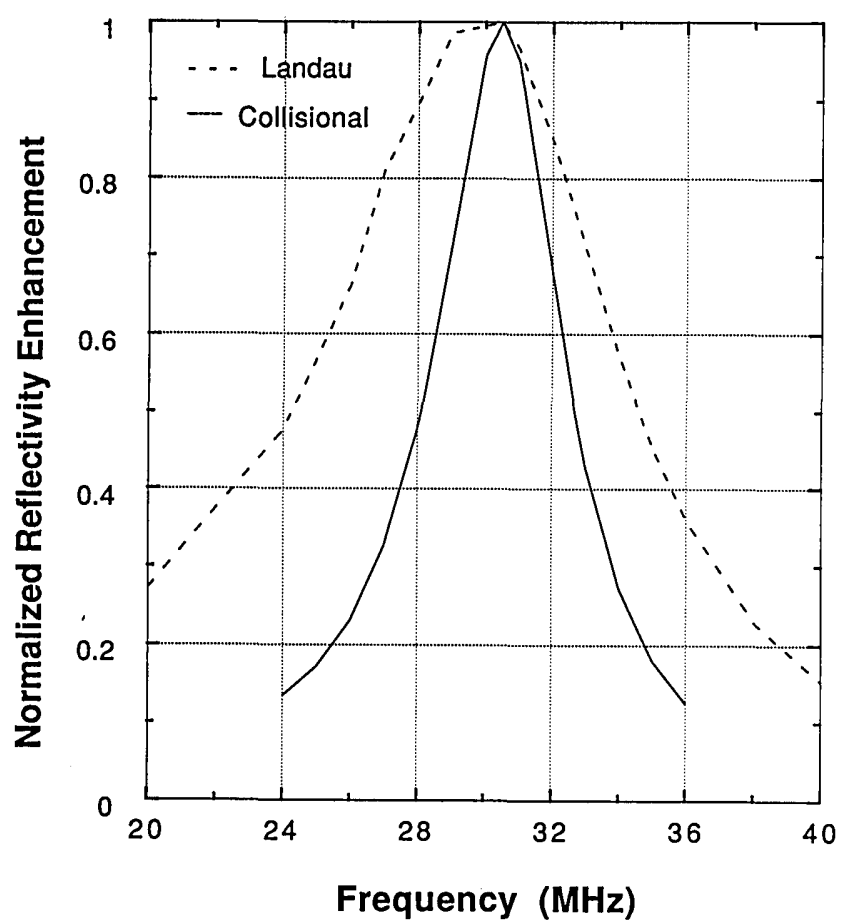

Fig. 4. Resonance profiles for Landau and collisional damping. If Landau damping is assumed to be the damping mechanism the profile is much broader. The two plots have been normalized to one, but the collisional peak is actually approximately 20 times larger than the Landau peak.

damping. Mostovych and DeSilva, ${ }^{12}$ and later Zhang et al., ${ }^{13}$ studied the thermal ion acoustic fluctuation spectrum of a collisional plasma and found that the widths of the resonance peaks are much narrower than the predictions of Landau damping.

A complete analysis of damping in the collisional regime has been performed by Federici. ${ }^{14}$ His analysis includes collisional damping, thermal damping, and viscous damping and is valid if the following conditions are satisfied:

$$
\begin{aligned}
w_{m n} & <v_{e}, \\
\frac{w_{f p} v_{e}}{w_{p i}{ }^{2}} & \ll 1, \\
\frac{v_{e} m_{e}}{w_{f p} m_{i}} & \ll 1 .
\end{aligned}
$$

These conditions are all met in our experiment. Assuming a temperature of $T_{e}=T_{i}=2.25 \mathrm{eV}$ and a density of $n_{0}=$ $9.5 \times 10^{16} \mathrm{~cm}^{-3}$, the important parameters are $w_{a c}=$ $1.92 \times 10^{8} \mathrm{sec}^{-1}, v_{e i}=2.89 \times 10^{11} \mathrm{sec}^{-1}$, and $w_{p i}^{2}=4.12 \times$ $10^{21} \mathrm{sec}^{-2}$. The damping rate in this analysis is approximately $\gamma=0.067 w_{a c}$. This is much smaller than Landau damping predicts, and fits fairly well with the experimentally measured width. A plot of normalized reflectivity versus frequency is shown in Fig. 4, for the cases of Landau damping and collisional damping.

\section{CONVECTION}

Although this model predicts the resonance width fairly well, it also predicts a large enhancement. Since the density increase is proportional to $w / \gamma$, this model should in- crease the density by a factor of 16 and the reflectivity by a factor of 256. However, the experimental reflectivity is enhanced by only a factor of 4 . There are many factors that can reduce the signal from the large predicted value. One important factor is the effects of convection. Although the plasma resonance increases the density modulation significantly, the propagating ion acoustic wave is also moving energy out of the plasma. Since the three beams interact in a 1-mm spot, there are only a few grating periods in the region. For the small- $k$ grating with a wave number $k_{f p}=413.74 \mathrm{~cm}^{-1}$, the grating wavelength is $\lambda_{f p}=2 \pi / k_{f p}=0.15 \mathrm{~mm}$. This means there are at most five or six grating periods in the region, which is not sufficient to allow the density modulation to build up to its peak value. In order to determine the density modulation, one must solve the differential equation with respect

(a)

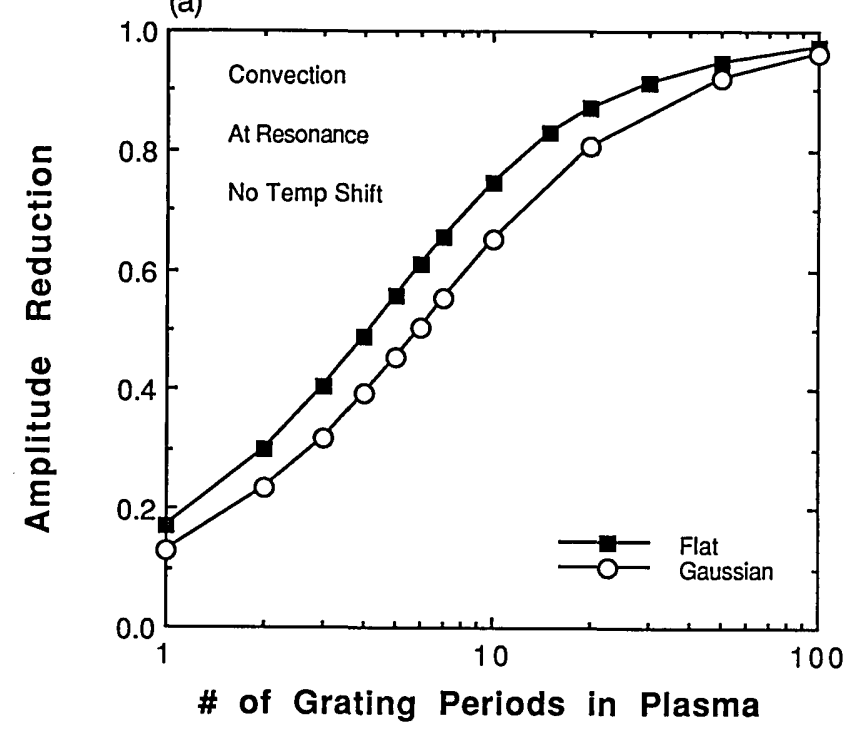

(b)

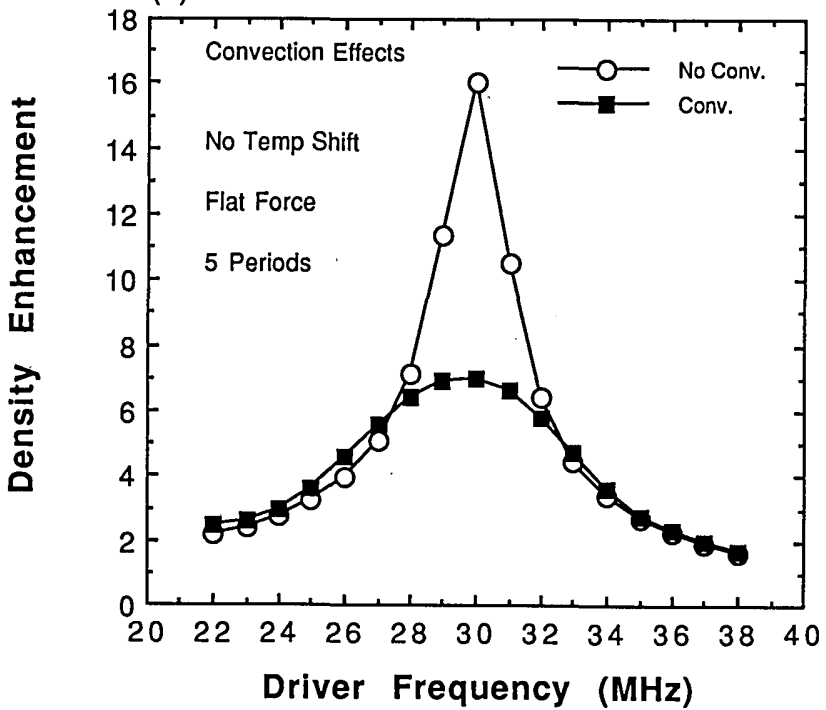

Fig. 5. The effects of convection are much more important at resonance than away from resonance. (a) Amplitude reduction at resonance as a function of the number of periods in the plasma. (b) Way in which the overall collisional damping profile is altered by convection, assuming approximately five grating periods in the plasma. 


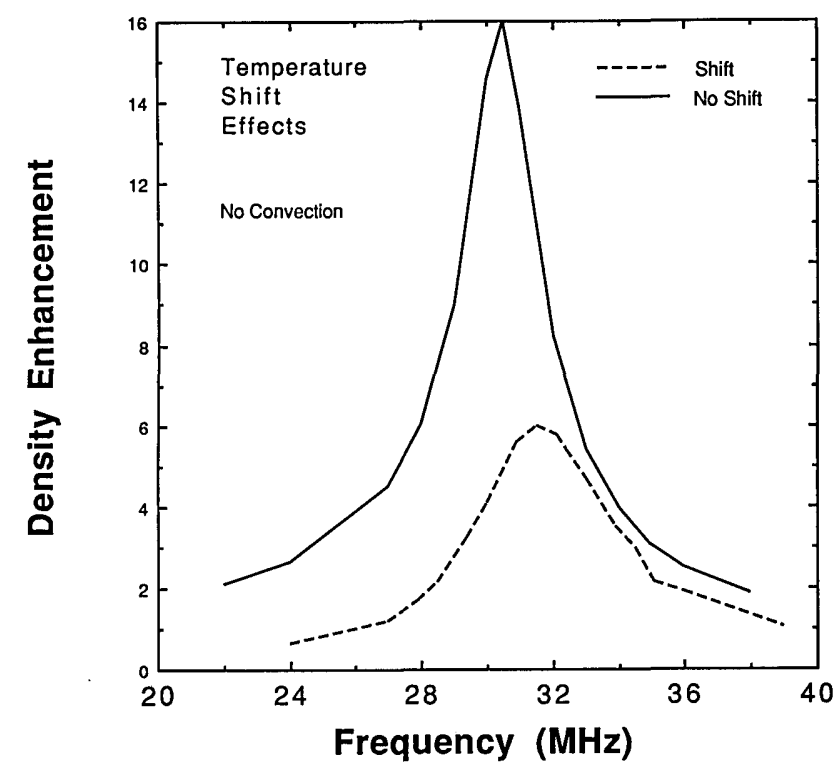

Fig. 6. During the duration of the laser pulse, the plasma temperature rises from 2 to approximately $5 \mathrm{eV}$. As a result the ion acoustic resonance is detuned by the changing temperature. This figure shows the original resonance profile and how it is affected by the laser heating. Convection effects are not considered here.

to space:

$$
\left(\frac{\partial^{2}}{\partial t^{2}}+\gamma \frac{\partial}{\partial t}+c_{s}^{2} \frac{\partial^{2}}{\partial x^{2}}\right) n(x)=\frac{j k}{M} F_{\mathrm{NL}} .
$$

The density profile can no longer be assumed to be constant in the $x$ direction. If the force is assumed to be uniform in the $x$ direction, then convection can reduce the density enhancement at resonance by a factor of approximately 3 . If a more realistic force is assumed, the reduction is even larger. Figure 5(a) shows the density reduction at resonance, as a function of grating periods in the plasma, for two cases: a flat force and a force with a Gaussian profile. Clearly, as the size of the plasma increases, convection becomes less and less important. In our case, however, with a plasma size of approximately $1 \mathrm{~mm}$, convection can reduce the reflectivity enhancement by one order of magnitude. Away from resonance, the effects of convection are not as important. Figure 5(b) shows how the Lorentzian profile is altered by convection.

\section{TEMPERATURE SHIFT}

The experimentally measured reflectivity is also reduced from the predicted value because of a temperature shift in the plasma. During the buildup of the laser pulse, the plasma electron temperature gradually begins to rise owing to collisional absorption, from 2 to approximately $5 \mathrm{eV}^{15}$ As a result, the plasma resonance is not present for the duration of the laser pulse. In the harmonic oscillator model, the density modulation $n_{1} / n_{0}$ can be solved from the second-order differential equation

$$
\left[\frac{\partial^{2}}{\partial t^{2}}+\gamma \frac{\partial}{\partial t}-k^{2} c_{s}^{2}(t)\right] n_{1}=\frac{j k}{M} F_{\mathrm{NL}} \cos w t .
$$

In this equation the ion acoustic velocity $w_{\mathrm{ac}}=k c_{s}$ now varies with time. The density modulation always requires a finite time to build up to its peak value. The ion acoustic velocity, however, changes before the density can reach its peak value. As a result, it can never be enhanced by the factor $w / \gamma$ as predicted. One must solve the differential equation, with a varying $w_{\mathrm{ac}}$, in order to determine the density modulation. A plot of the peak density modulation $n_{1} / n_{0}$, including the temperature shift, versus applied frequency is shown in Fig. 6. The changing resonance frequency may also explain the sharpness of the signal pulse shape (see Fig. 2), since the resonance is not present for the duration of the incident pulse. One possible way to reduce the detuning effects of laser heating is to reduce the laser intensity. If it is assumed, however, that the plasma heating occurs by inverse bremsstrahlung and the heat loss occurs by radial convection, then the heated plasma temperature has a weak dependence on laser power. Consequently, it was not possible in the present experiment to eliminate the heating effects by simply reducing the laser intensity.

To include the effects of convection and the temperature shift, one must solve the second-order differential equation with respect to both time and space. Each effect working alone can reduce the density modulation by a factor of approximately 3 . If both processes are considered, however, it is not yet known whether the two effects will work together. Figure 3(a) shows a simple attempt to include both convection and temperature shift effects. The differential equation in space was solved first with convection effects only. For a given driver frequency, the reduction by convection was determined for different ion acoustic frequencies. The differential equation in time was then solved with temperature shift effects. As the ion acoustic frequency changed, the driver force was reduced by the appropriate convection factor. At resonance, the driver force was reduced by a factor of 3 , while far away from resonance the force was unaffected. Figure 3(a) shows the reflectivity versus applied driver frequency when the above procedure is used. (The solid curve is the theoretical prediction.) The plot shows good agreement with the experimental data for the peak reflectivity. Far away from resonance, however, the experimental data do not fit quite so well, since the measured reflectivity remains somewhat higher than this model predicts. This discrepancy may occur because the model assumes singlefrequency laser beams. Experimentally, however, the pump has some component at $w_{0} \pm w_{m}$, so the reflectivity always has some DFWM level. A more complete analysis involves solving for the density modulation as a function of space and time simultaneously, including both convection and laser heating effects.

In the above discussion, no account is made of either the reflectivity or the spectral width for spatial temperature and density inhomogeneities. This is because the wave mixing is carried out in a preformed plasma that has a size much greater than the interaction length. In other circumstances, inhomogeneities can be quite important. ${ }^{16}$

\section{CONCLUSION}

The DFWM experiments clearly show that the plasma is in the collisional regime. The importance of the thermal force can be seen by the dominance of the small- $k$ grating. 
Also, the reflectivity scales as $n_{0}{ }^{6}$, which indicates the thermal force is the main nonlinear mechanism.

In RFWM the reflectivity shows enhancement at the ion acoustic frequency. When the gas is changed, the resonance frequency shifts because the ion acoustic velocity changes. The measured resonance frequency varies as $1 / \sqrt{M}$, as the theory predicts. From the width of the resonance curve it is clear that collisional damping is the important damping mechanism. The enhancement, however, is not so large as expected, owing to the effects of convection and the temperature shift. If these effects could be reduced, by increasing the plasma size and decreasing the laser heating, then RFWM could be a useful diagnostic for determining the plasma temperature, the ion acoustic velocity, and the damping rate.

\section{ACKNOWLEDGMENTS}

This work was supported by the Lawrence Livermore National Laboratory University Research Program, U.S. Department of Energy contract DE-AO3-83-ER40120 and University of California CALCOR funds. We thank W. Mori, Y. Kitagawa, and C. Clayton for many useful discussions.

\section{REFERENCES}

1. R. A. Fisher, ed., Optical Phase Conjugation (Academic, New York, 1983).

2. D. M. Pepper, "Nonlinear optical phase conjugation," IEEE J. Quantum Electron. 25, 312 (1989).

3. D. M. Pepper, "Nonlinear optical phase conjugation," in
Laser Handbook M. L. Stitch and M. Bass, eds. (Elsevier, New York, 1985), Vol. 4.

4. D. Steel and J. Lam, "Degenerate four wave mixing in plasmas," Opt. Lett. 4, 363-365 (1979).

5. J. Federici and D. K. Mansfield, "Degenerate four wave mixing and phase conjugation in a collisional plasma," J. Opt. Soc. Am. B 3, 1586-1595 (1986).

6. Y. Kitagawa, R. Savage, and C. Joshi, "Demonstration of collisionally enhanced degenerate four wave mixing in a plasma," Phys. Rev. Lett. 62, 151-154 (1989).

7. I. Nebenzahl, A. Ron, and N. Rostoker, "Reflected phase conjugate wave in a plasma," Phys. Rev. Lett. 60, 1030-1032 (1988).

8. M. V. Goldman and E. A. Williams, "Time dependent phase conjugation and four wave mixing in plasmas," Phys. Fluids B 3, 751-765 (1991).

9. G. Baym and R. W. Hellwarth, "Nonlinear optics of many particle systems," IEEE J. Quantum Electron. QE-1, 309320 (1985).

10. T. Lehner, "Longitudinal plasma resonances excited by optical mixing and detected by phase conjugation," Phys. Scr. 39, 595-605 (1989).

11. F. F. Chen, Introduction to Plasma Physics (Plenum, New York, 1985), Vol. 1.

12. A. Mostovych and A. DeSilva, "Laser scatter measurements of thermal ion fluctuations in collisionally dominated plasmas," Phys. Rev. A 34, 3238-3252 (1986).

13. Y. Q. Zhang, A. W. DeSilva, and A. N. Mostovych, "Density fluctuation spectra of a collision-dominated plasma measured by light scattering," Phys. Rev. Lett. 62, 1848-1851 (1989).

14. J. Federici, "Four wave mixing and phase conjugation in plasmas," Ph.D. dissertation (Princeton University, Princeton, New Jersey, 1988).

15. F. F. Chen, "Laser heating of underdense magnetized plasmas," UCLA Rep. 34-P157 (University of California, Los Angeles, 1985).

16. E. Williams, D. Lininger, and M. Goldman, "Phase conjugation by four wave mixing in inhomogeneous plasmas," Phys. Fluids B 1, 1561-1564 (1989). 